\title{
Acciones gubernamentales para la detección temprana del cáncer de mama en América Latina. Retos a futuro
}

\author{
Luz María González-Robledo, PhD, (') María Cecilia González-Robledo, MC,(I) \\ Gustavo Nigenda, PhD, (1) Lizbeth López-Carrillo, PhD. (2)
}

\author{
González-Robledo LM, González-Robledo MC, \\ Nigenda G, López-Carrillo L. \\ Acciones gubernamentales para la detección temprana \\ del cáncer de mama en América Latina. Retos a futuro. \\ Salud Publica Mex 2010;52:533-543.
}

\begin{abstract}
Resumen
Se presenta investigación documental realizada en 2009 que pretende documentar el marco normativo y los programas vigentes para la detección temprana del cáncer mamario en América Latina y establecer los retos más importantes para contener la epidemia en la región. Se identificaron diversas iniciativas emprendidas por las autoridades gubernamentales de salud para contrarrestar el crecimiento de la morbimortalidad por esta causa mediante estrategias de detección temprana, atención e investigación. A pesar de los avances en el diseño de políticas, programas, protocolos y guías de manejo clínico, persisten importantes retos para lograr los objetivos propuestos y la contención de este problema.
\end{abstract}

Palabras clave: políticas públicas de salud; cáncer de mama; América Latina y el Caribe

\author{
González-Robledo LM, González-Robledo MC, \\ Nigenda G, López-Carrillo L. \\ Government actions for the early detection \\ of breast cancer in Latin America. Future challenges. \\ Salud Publica Mex 2010;52:533-543.
}

\begin{abstract}
Documentary research carried out in 2009 aims to document the regulatory framework and existing programs for the early detection of breast cancer in Latin America and the Caribbean in order to establish the most important challenges for the containment of the epidemic in the region. The governments of the region have developed diverse efforts and initiatives to confront the rise in mortality due to said cause, including early detection, treatment and research strategies. Despite advances in the early detection of breast cancer, the challenge remains to link efforts to ensure continuity of care (diagnostic confirmation, treatment and monitoring) in order to achieve higher efficiency, effectiveness and benefits for women with this disease.
\end{abstract}

Keywords: health public policy; breast cancer; Latin America and the Caribbean
A ctualmente, el cáncer de mama (CaMa) es un problema de salud pública no reconocido como prioritario por la mayoría de las autoridades de salud en la región de las Américas, pese al continuo incremento de las tasas de incidencia y mortalidad. ${ }^{1-3}$ En América
Latina se constituye como la primera causa de muerte por neoplasias malignas femeninas, desplazando en diversos países al cáncer cervicouterino., 1,4,5

De acuerdo con la regionalización de la Agencia Internacional para la Investigación en Cáncer (IARC, por

(I) Centro de Investigación en Sistemas de Salud. Instituto Nacional de Salud Pública. Cuernavaca, Morelos, México.

(2) Centro de Investigación en Salud Poblacional. Instituto Nacional de Salud Pública. Cuernavaca, Morelos, México.

Fecha de recibido: 13 de abril de 2010 - Fecha de aceptado: 7 de septiembre de 2010 Solicitud de sobretiros: Dra. Luz María González Robledo. Instituto Nacional de Salud Pública. Av. Universidad 655, Col. Santa María Ahuacatitlán. 62100 Cuernavaca, Morelos, México.

Correo electrónico: luz.gonzalez@insp.mx 
sus siglas en inglés) en 2002, la tasa más alta de CaMa por 100 mil mujeres en América Latina y El Caribe (ALC) se presentó en Sudamérica con 46 casos por 100 mil mujeres, seguida por El Caribe con 32.9 y Centroamérica con 25.9, y son Argentina y Uruguay, en el cono sur, los países que presentan las mayores tasas de incidencia con 75 y $83 \mathrm{ca}$ sos. Cifras similares se observaron, en el mismo período, en Estados Unidos de América y Canadá. ${ }^{1}$

Aunque persisten dificultades para obtener datos actualizados y confiables sobre incidencia y letalidad por CaMa en algunos países de ALC, las estadísticas de mortalidad por esta causa en la mayoría de estos países van en aumento, particularmente en Colombia, Costa Rica, Ecuador, México y Venezuela que hasta recientemente contaban con tasas relativamente bajas. ${ }^{6}$ En contraste, Canadá y Estados Unidos de América han presentado un descenso reciente de la mortalidad por esta causa, asociada no sólo a la efectividad de los programas de detección temprana, sino también a la oportunidad y calidad del tratamiento basado en guías de práctica clínica, incorporación oportuna de nuevas tecnologías, educación continua del personal de salud y fomento a protocolos de investigación. ${ }^{7}$

En México, a partir de 2006 el CaMa constituye la primera causa de muerte por patología maligna en mujeres mayores de 25 años, superando la mortalidad por cáncer cervicouterino. ${ }^{8,9}$ Un dato relevante es el hecho de que en México más de 75\% de los casos son diagnosticados en etapas localmente avanzadas de la enfermedad (II, III y IV) de acuerdo con datos del Registro Histopatológico de Neoplasias Malignas (RHNM). ${ }^{10}$

Actualmente, existe consenso en la literatura científica de que una de las medidas más efectivas para reducir la mortalidad por CaMa es la prevención secundaria. La detección temprana y el diagnóstico en estadios más iniciales mejoran el pronóstico de la enfermedad.

Bajo este contexto resulta importante conocer el enfoque de las acciones gubernamentales de países de América Latina tendientes a enfrentar el crecimiento del CaMa en su población, así como analizar aspectos de su aplicación tanto en términos legislativos como operativos, e identificar aciertos y retos. Debido a la extensión de los temas relacionados con la respuesta de los sistemas de salud al cáncer de mama, este artículo se centra en el tema de la detección temprana sin que esto implique demérito de la importancia de otras áreas de enfoque de las acciones gubernamentales como el diagnóstico o el tratamiento.

El estudio se basó en una revisión de literatura realizada por los autores entre agosto de 2008 y abril de 2009 bajo los fundamentos de la investigación documental. La búsqueda de documentos fue realizada principalmente en medios electrónicos: bases de datos
(PubMed / Medline, Lilacs, Scielo y Google Scholar) y páginas electrónicas de los ministerios y secretarías de salud de los países de América Latina (AL). La búsqueda se restringió a la literatura publicada entre 1990 a 2008 y en los idiomas español, inglés y portugués (para el caso de Brasil).

El criterio utilizado para seleccionar la información fue el de limitar la búsqueda a todas aquellas publicaciones que relacionaran en sus resúmenes, títulos o textos a las siguientes palabras claves (descriptores): programa, políticas, planes, norma, reglamento, ley, resolución, guía, detección temprana, tamizaje, mamografía, además, cáncer de mama y cada uno de los países de América Latina (Argentina, Bolivia, Brasil, Chile, Colombia, Costa Rica, Cuba, Ecuador, El Salvador, Guatemala, Honduras, México, Nicaragua, Panamá, Paraguay, Perú, Uruguay, Venezuela).

Los resultados de la búsqueda arrojaron un listado de 826 documentos entre los cuales se destacan programas, protocolos, guías de atención, manuales, documentos informativos publicados por los ministerios y secretarías de salud tanto en el ámbito nacional como local, leyes, normas, reglamentos e informes emitidos por órganos del gobierno, comisiones especiales, consejos nacionales y agencias internacionales como OMS/OPS, así como artículos de revisión y estudios originales relacionados con la temática propuesta. Del total de documentos encontrados, fue posible ubicar 358 y de éstos 90 aportaron información útil para esta revisión, a partir de cumplir con los criterios de inclusión propuestos por el equipo de investigación: instrumentos jurídico-normativos que incluyeran acciones de prevención secundaria de cáncer de mama (Leyes, Reglamentos, Decretos, Resoluciones, Normas y Ordenanzas) y documentos, artículos e informes que abarcaran lineamientos de política, programas, planes y estrategias para la detección y diagnóstico del cáncer de mama. Se excluyeron 268 documentos por no cumplir con los criterios de inclusión (no correspondían a la temática investigada, eran artículos de opinión, el contenido versaba sobre tratamientos).

Las categorías que guiaron la identificación y análisis de la información se centraron en el marco jurídiconormativo, programas y planes y otros mecanismos (protocolos, guías y consensos de manejo clínico) para la detección y diagnóstico de cáncer de mama los cuales se toman como base para la presentación de los resultados. Los aspectos explorados incluyeron el nombre o denominación del programa/plan/guía/normativa, institución responsable, instituciones participantes, ámbito de aplicación (local, provincial/estatal, nacional), año de creación/implementación, población a la que va dirigido, objetivos, actividades y/o acciones que 
contempla el programa, edad en la que está planteado iniciar el tamizaje mamográfico y periodicidad de la prueba y resultados obtenidos, así como resultados de las acciones emprendidas.

\section{Detección temprana de cáncer de mama}

En términos generales, la detección temprana se enfoca en descubrir y curar las condiciones que producen un efecto patológico sin que necesariamente se presenten signos y síntomas visibles de la afección. Para el caso específico del CaMa, existen diversos mecanismos para detectar la enfermedad como son la autoexploración, la exploración clínica mamaria y el tamizaje mamográfico a fin de identificarlo en estadios mucho más tempranos. ${ }^{11-14}$ Múltiples estudios recomiendan desarrollar programas de tamizaje mamográfico para reducir significativamente la morbi-mortalidad del CaMa, dado que permite diagnosticar la patología en etapas donde aún no se presentan signos y síntomas, lo cual mejora de manera importante el pronóstico de la enfermedad.

El tamizaje es una estrategia empleada para detectar enfermedades precozmente. Consiste en llevar a cabo una selección de individuos que pueden estar enfermos o en riesgo de padecer una determinada enfermedad de los que no lo están. ${ }^{15}$ Existen dos tipos de tamizaje para la detección del CaMa: tamizaje poblacional y tamizaje de oportunidad. El primero opera como programa organizado de salud pública promovido generalmente por las autoridades de salud. Implica la aplicación de pruebas a grandes volúmenes de población considerada en riesgo, y tienen como meta el descenso de la mortalidad. El segundo sucede fuera de un programa formal, y en éste el proveedor de salud envía una mujer sin síntomas a realizarse una prueba. Ambas estrategias deben asegurar la valoración diagnóstica de las mujeres en caso de que se descubran anormalidades y, más aún, el acceso a métodos terapéuticos y de seguimiento adecuados y pertinentes. ${ }^{16-18}$

La efectividad y los beneficios del tamizaje del CaMa mediante mamografía han sido ampliamente estudiados en diferentes países del mundo, a través de ensayos aleatorios controlados y meta-análisis. Los resultados han permitido demostrar reducción de la mortalidad variable (hasta de 30\%) según la edad, los años de seguimiento, la adherencia al programa, el número de mujeres y la periodicidad de la mamografía. ${ }^{19-23}$ Los mayores beneficios se reportan en mujeres entre los 50 y 69 años. ${ }^{24-26}$

\section{Acciones gubernamentales para la detección temprana y diagnóstico oportuno del cáncer de mama}

En general, la estrategia pública para abordar la problemática del cáncer de mama en AL opera a través de la regulación, el diseño e implementación de programas de detección temprana y la provisión de atención a través de la red de prestadores de servicios públicos y privados. Los diversos gobiernos de la región han desarrollado múltiples esfuerzos para contrarrestar el rápido crecimiento de las tasas de mortalidad mediante diversos mecanismos los cuales hemos agrupado en tres categorías: a) marco legal y normativo, b) programas y planes para la detección temprana y, c) otros mecanismos. Esta última categoría incluye aquellos instrumentos que no fue posible tipificar exclusivamente en alguna de las dos primeras categorías en virtud de que no ostentan un carácter jurídico o no son propiamente programas.

\section{Marco jurídico-normativo}

El cuadro I resume los principales hallazgos en materia legal y normativa que sustenta las políticas, programas y estrategias para la detección temprana del cáncer de mama en América Latina.

En toda la región, el desarrollo del marco regulatorio para la atención del cáncer de mama ha progresado notoriamente. En la mayoría de países de AL se cuenta con un conjunto de normas, leyes, decretos y reglamentos que establecen con precisión las acciones e intervenciones para la detección temprana, diagnóstico, tratamiento y seguimiento de la población con la patología. Esta normatividad también establece en algunos casos: a) la obligatoriedad en la observancia de las acciones del programa para todos los prestadores de servicios de salud, público, social y privado; b) estandarización de los procedimientos técnico-administrativos para la atención del CaMa, c) los mecanismos de referencia y contrarreferencia para la atención, d) los sistemas de información y registro, e) criterios para el control de calidad de la atención y f) los criterios para la vigilancia epidemiológica de la enfermedad.

\section{Programas y planes}

La instrumentación y operación del marco regulatorio, así como de las políticas en materia de CaMa, se llevan a cabo a través del desarrollo de programas y planes de acción específicos. La revisión evidenció en este aspecto los hallazgos que se describen en el cuadro II. 


\section{Cuadro I}

\section{Marco normativo para la atención del cáncer de mama en América Latina, 2009}

\begin{tabular}{|c|c|c|c|c|c|}
\hline \multirow[b]{2}{*}{ País } & \multirow[b]{2}{*}{ Norma/regulación } & \multicolumn{4}{|c|}{ Acciones contempladas } \\
\hline & & 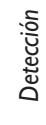 & 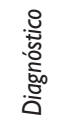 & 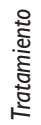 & 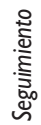 \\
\hline Argentina $a^{54-56}$ & $\begin{array}{l}\text { Resolución Secretarial N 2886/76. Programa Nacional de Control de Cáncer } \\
\text { Resolución ministerial N59/03. Sub-programa de prevención secundaria del cáncer } \\
\text { de Mama. } \\
\text { Resolución 939/2000 del Ministerio de Salud. Programa Médico-Obligatorio (PMO). } \\
\text { Incluye Programas de Prevención para el Cáncer de Mama y de Cuello de Utero. } \\
\text { Régimen de Asistencia Obligatoria, para todas las Obras Sociales del sistema de la } \\
\text { Ley } 23.660 \text { y 23.66I } \\
\text { Ley } 25.673 \text { y decreto reglamentario I282/2003 (B. O.26/05/2003).Programa Nacional } \\
\text { de Salud Sexual y Procreación Responsable. Ministerio de Salud }\end{array}$ & $x$ & $x$ & $x$ & $x$ \\
\hline Brasil $^{57}$ & $\begin{array}{l}\text { Decreto } n^{\circ} \text { 2607/2004. Plan Nacional de Salud - Un Pacto por la salud en el Brasil. } \\
\text { PT n } 2439 \text { / GM de } 8 \text { de diciembre de 2005. Política Nacional de Oncología. }\end{array}$ & $x$ & $x$ & $x$ & $x$ \\
\hline Bolivia $^{30}$ & $\begin{array}{l}\text { Resolución Ministerial } 0903 \text { del } 20 \text { de diciembre de 2004.Plan de prevención y control } \\
\text { de enfermedades no transmisibles para la gestión 2005-2009. }\end{array}$ & $x$ & $x$ & $x$ & $x$ \\
\hline Chile $^{58,59}$ & $\begin{array}{l}\text { Ley n } 19.966 \text { Régimen General de Garantías en Salud. Decreto Supremo No. } 44 \\
\text { del } 9 \text { de enero de } 2007 .\end{array}$ & $x$ & $x$ & $x$ & $x$ \\
\hline Colombia ${ }^{60-62}$ & $\begin{array}{l}\text { Resolución nº 004I2 DE 2000. Norma Técnica para la Detección Temprana de } \\
\text { Cáncer de Seno }\end{array}$ & $x$ & $x$ & $x$ & $x$ \\
\hline Ecuador $^{33}$ & $\begin{array}{l}\text { Conjunto de Prestaciones del Sistema Nacional de Salud, Resolución del Directorio } \\
\text { del Consejo Nacional de Salud del } 25 \text { de octubre del 2006; Acuerdo Ministerial } \\
n^{\circ} 0000620 \text { del I } 2 \text { de enero de } 2007 \text { y Acuerdo Ministerial n } 0000116 \text { del } 16 \text { de } \\
\text { marzo de } 2007\end{array}$ & $x$ & $x$ & & \\
\hline México $^{63}$ & $\begin{array}{l}\text { Norma Oficial (NOM-04I-SSA2-2002) para la prevención, diagnóstico, tratamiento, } \\
\text { control y vigilancia epidemiológica del cáncer de mama. }\end{array}$ & $x$ & $x$ & $x$ & \\
\hline Panamá64 & $\begin{array}{l}\text { Norma de Atención Integral de la Mujer. Componente Detección del cáncer de } \\
\text { mamas. }\end{array}$ & $x$ & $x$ & $x$ & \\
\hline Perúu ${ }^{65}$ & $\begin{array}{l}\text { Resolución Jefatural } \mathrm{n}^{\circ} \mathrm{I} 2 \mathrm{I} \text { abril de } 2008 \text {. } \\
\text { Norma técnico-oncológica para la prevención, detección y diagnóstico temprano } \\
\text { del cáncer de mama a nivel nacional. }\end{array}$ & $x$ & $x$ & $x$ & $x$ \\
\hline Uruguay ${ }^{66}$ & $\begin{array}{l}\text { Decreto del Poder Ejecutivo del } n^{\circ} \text { 202/005, 27/06/2005. Programa Nacional de } \\
\text { Control de Cáncer (PRONACCAN) }\end{array}$ & $x$ & $x$ & $x$ & $x$ \\
\hline
\end{tabular}

Un aspecto común identificado en la región fue que las acciones de prevención secundaria del CaMa han formado parte de programas de salud integrados más amplios, dirigidos particularmente a la salud de la mujer. Se han agrupado primordialmente con programas de prevención y control del cáncer cervicouterino ( $\mathrm{CaCu})$. Algunos de estos casos se encuentran en Argentina (Programas de detección precoz del cáncer génito-mamario y enfermedades de transmisión sexual -PRODEGEM-; Programa Nacional de Salud Sexual y Procreación), ${ }^{27,28}$ Brasil (Programa Nacional de Control de Cáncer de cuello de útero y de mama -Viva Mulher-), ${ }^{29}$ Bolivia (Prevención y control de enfermedades no transmisibles en la atención primaria de salud), ${ }^{30}$ Uruguay (Programa
Nacional para el Control del Cáncer), ${ }^{31}$ Ecuador (Conjunto de Prestaciones del Sistema Nacional de Salud y Políticas integrales de Salud para el Adulto, Adulto Mayor), ${ }^{32,33}$ Perú (Plan Nacional para el fortalecimiento de la prevención y control del cáncer en el Perú) ${ }^{34}$ y El Salvador (Gerencia de Atención integral en salud a la mujer y la niñez). ${ }^{35}$

A partir del ingreso del CaMa a las prioridades programáticas en materia de salud de algunos gobiernos de AL, en muchos de los países se han desarrollado programas específicos para la detección temprana de la enfermedad. ${ }^{36-40}$ México mantuvo integrado el componente de CaMa al programa de CaCu durante más de 25 años (Programa Nacional de Detección Oportuna 


\section{Cuadro II}

\section{Programas y planes para la detección temprana del Cáncer de mama en Ámerica latina, 2009}

País

\begin{tabular}{ll} 
& $\begin{array}{l}\text { Programa de Detección Precoz de Cáncer Genito- } \\
\text { Mamario (PROGEMA) }\end{array}$ \\
\cline { 2 - 2 } Argentina $^{67,68}$ & $\begin{array}{l}\text { Programa de Enfermedades Oncológicas (PMO) } \\
\text { Incluyen: los programas de prevención de los cán- } \\
\text { ceres femeninos, mama y cuello uterino. }\end{array}$ \\
\hline Brasil ${ }^{57,69}$ & $\begin{array}{l}\text { Programa Nacional de Control de Cáncer de cuello } \\
\text { de útero y de mama "Viva Mulher" }\end{array}$
\end{tabular}

Programa de Detección Precoz de Cáncer Genito-

Incluyen: los programas de prevención de los cánPrograma Nacional de Control de Cán "Viva Mulher"

Bolivia $^{30}$

Plan de prevención y control de enfermedades no transmisibles para la gestión 2005-2009.
- Mamografía de base a partir de los 35 años y 30 años con antecedentes directos de cáncer mamario (madre, hermanas).

- Mamografía de control anual mujeres de 50 años y más.

- Mamografía de control bianual a mujeres entre 40 y 49 años (dependiendo del riesgo).
Mamografía de tamizaje anual a mujeres de 35 años pertenecientes a grupos de alto riesgo para cáncer de mama, y a todas las mujeres de 40 años y más.

- Autoexamen de mama.

- Examen clínico periódico, por el médico de asistencia.

- Mamografía de tamizaje a mujeres mayores de 40 años anual o bianual (depende del riesgo)

- Mamografía de diagnóstico para confirmar una lesión sospechosa o no detectable.

Chile $^{70} \quad$ Programa Nacional de Cáncer de Mama

\begin{tabular}{ll}
\hline México $^{71}$ & Programa de Acción 2007-20I2 Cáncer de mama \\
\hline Panamá $^{72}$ & Programa de salud integral de la mujer \\
\hline Perú & \\
\hline & $\begin{array}{l}\text { Plan Nacional para el fortalecimiento de la preven- } \\
\text { Control del cáncer de mama }\end{array}$ \\
\hline & $\begin{array}{l}\text { Programa de Detección Precoz de Cáncer de } \\
\text { Mama }\end{array}$
\end{tabular}

Fuente: Elaboración propia de los autores con información de documentos oficiales publicados por cada país
Mamografía de detección para toda mujer de 50 años. Cuando existe sospecha por examen clínico se le solicita una mamografía y si ésta es informada como sospechosa, la persona es referida al nivel secundario de atención.

- Autoexploración mamaria: se contempla la capacitación a las mujeres de 20 años de edad y más.

- Exploración clínica de mama: se ofrece este servicio para mujeres de 25 y más años de edad, con una periodicidad anual.

- Mastografía para la detección de acuerdo con la normatividad, focalizando en las mujeres de 50-69 años de edad y de 40 años cuando tienen antecedentes familiares.

- Autoexploración mamaria: capacitación a todas las mujeres.

- Exploración clínica de mama.

- Mastografía para la detección a cualquier edad: si existen signos clínicos sospechosos de cáncer, base a partir de los 35 años, cada I-2 años: mujeres entre los 40 y 50 años, cada año: a partir de los 50 años de edad.

- Autoexamen de mama: Educar a las mujeres mayores de 20 años.

- Identificar a las mujeres de riesgo y referirlas para tamizaje de cáncer de mama

- Mamografía: se realiza al 30\% de la población de mujeres mayores de 40 años.

La población objetivo del Programa de Cáncer Mamario (PCM) del Ministerio de Salud se limita por el momento a realizar el examen físico mamario e involucra a mujeres entre 20 y 39 años cada tres años y anual a partir de los 40 años.

El programa de detección oportuna de cáncer de mama es desarrollado por la Comisión Honoraria de Lucha contra el Cáncer (CHLCC) en todo el país. Se realizan mamografías a todas las mujeres mayores de 40 años que las soliciten de Cáncer cervicouterino y cáncer de mama). En la actualidad, las instituciones de seguridad social, Instituto Mexicano del Seguro Social (IMSS) y el Instituto de Seguridad y Servicios Sociales de los Trabajadores del Estado (ISSSTE), mantienen el componente de cáncer de mama dentro de los programas "Modelo de Atención Integral a la Salud Ginecológica"41-42 y "Programa Institucional de Atención a Neoplasias" ${ }^{43}$ respectivamente. A raíz del incremento de la morbi-mortalidad por CaMa, las autoridades de salud mexicanas deciden, en 2002, separar los dos componentes ( $\mathrm{CaCu}$ y $\mathrm{CaMa}$ ) en programas específicos. ${ }^{54}$ 


\section{Cuadro III}

\section{Protocolos y guías para la detección temprana del Cáncer de mama en AL, 2009}

País

\begin{tabular}{ll} 
Argentina $^{74,75}$ & $\begin{array}{l}\text { Consenso Nacional Inter-Sociedades } \\
\text { sobre Cáncer de Mama: Pautas para el } \\
\text { Diagnóstico y Manejo de las Lesiones } \\
\text { Mamarias Subclínicas. }\end{array}$ \\
\hline Brasil & $\begin{array}{l}\text { Control de Cáncer de Mama. Documento } \\
\text { de Consenso. }\end{array}$ \\
& $\begin{array}{l}\text { Controle dos Cânceres do Colo do } \\
\text { Utero e da Mama. Cadernos de Atencao } \\
\text { Básica. }\end{array}$
\end{tabular}

Chile ${ }^{76}$

Guía Clínica
- Mujeres a partir de los 40 años. Periodicidad anual.

- Mujeres con antecedentes familiares a partir de los 35 años o 10 años antes del familiar más joven con cáncer de mama. Periodicidad anual.

- Mujeres con tratamiento hormonal sustituto, de cualquier edad.

- Mamografía para mujeres de 50 a 69 años de edad, con un intervalo máximo de dos años entre los exámenes.

- Mamografía anual para mujeres a partir de los 35 años con alto riesgo para cáncer de mama

I. Mamografía y Examen Físico de Mama (EFM) a mujeres de 40 años y más, con uno o más factores de riesgo.

2. Mamografía y EFM a mujeres previo inicio de terapia hormonal de reemplazo.

3. Mamografía y EFM a todas las mujeres sintomáticas de 30 años y más.

4. Mamografía a todas las mujeres sintomáticas menores de 30 años con examen físico de mama sospechoso de probable patología maligna.

5. Mujeres de 50 años con mamografía sospechosa.

I. En Atención primaria en salud todo caso sospechoso de probable patología maligna debe realizarse la Solicitud de mamografía.

Chile* Protocolo AUGE

2. Los casos sospechosos por examen clínico de mama $(+)$, por mamografía y/o por ecotomografía se derivan a segundo nivel de atención para confirmación diagnóstica.

\begin{tabular}{|c|c|c|}
\hline Colombia $^{53}$ & $\begin{array}{l}\text { Guía de práctica clínica: Recomendacio- } \\
\text { nes para la detección temprana del cáncer }\end{array}$ & $\begin{array}{l}\text { - Tamizaje de oportunidad periódica, en mujeres asintomáticas mediante mamografía } \\
\text { a mujeres de } 50 \text { a } 69 \text { años. }\end{array}$ \\
\hline
\end{tabular}
nes para la detección temprana del cancer a mujeres de 50 a 69 años.

de mama en Colombia

- Mujer con riesgo promedio: mamografía anual iniciando a los 40 años.

\begin{tabular}{lll}
\hline Costa Rica ${ }^{77}$ & $\begin{array}{l}\text { Normas y procedimientos para el trata- } \\
\text { miento del cáncer en } \\
\text { Costa Rica (2009) }\end{array}$ & $\begin{array}{l}\text { - Mujer con riesgo promedio: mamografía anual iniciando a los } 40 \text { años. } \\
\text { Mujer con historia familiar de cáncer de mama/ovario mamografía anual iniciando I0 } \\
\text { años antes del familiar afectado pero no antes de los } 25 \text { años. } \\
\text { Linfoma de Hodgkin tratado con radiación: mamografía anual iniciando } 8 \text { años después } \\
\text { del tratamiento. }\end{array}$ \\
\hline México $^{71}$ & $\begin{array}{l}\text { Guía Técnica PREVENIMSS Mujeres de } \\
20 \text { a } 59 \text { años }\end{array}$ & Mamografía bianual a partir de los 50 años hasta los 69 años.
\end{tabular}

- Nivel primario de atención: identificación de grupos de riesgo y ante sospecha de patología mamaria maligna, se referirá al segundo nivel de atención.

$\begin{array}{ll}\text { El Salvador } & \text { Guía Técnica de Prevenciór } \\ & \text { Cáncer de Mama } 2006\end{array}$

- En el nivel secundario: diagnóstico definitivo de los casos sospechosos (mamografía, ultrasonido, patología, etc), derivación de los casos con diagnóstico de cáncer a la Unidad de Patología Mamaria del tercer nivel de atención (servicio de cirugía oncológica o ginecología oncológica).

Mamografía en los siguientes casos:

a) Mujeres de 40 años y más cada uno o dos años.

\begin{tabular}{|c|c|c|}
\hline Uruguay ${ }^{66}$ & $\begin{array}{l}\text { Manual de oncología para el primer nivel } \\
\text { de atención }\end{array}$ & $\begin{array}{l}\text { b) Mujeres que tienen un riesgo mayor que el promedio de padecer cáncer de mama } \\
\text { deberán hablar con su médico acerca de los controles mamográficos, momento de } \\
\text { inicio y frecuencia. } \\
\text { c) Mujeres de cualquier edad con hallazgo clínico sospechoso. }\end{array}$ \\
\hline
\end{tabular}

Fuente: Elaboración propia de los autores con información de documentos oficiales publicados por cada país

*Ministerio de Salud. Protocolo AUGE. Diagnóstico y Tratamiento Cáncer de Mama. Documento de Trabajo. Chile: Ministerio de Salud, 2004

\section{Otros mecanismos}

Complementariamente a la regulación y a los instrumentos de política pública, se ha desarrollado otra serie de iniciativas surgidas desde diferentes ámbitos institucionales, incluidas en muchos casos las de organizaciones no gubernamentales o privadas (colegios profesionales, agrupaciones científicas, organizaciones 
de la sociedad civil dedicadas al tema, entre otros). Estas iniciativas corresponden, en su mayoría, a directrices elaboradas sistemáticamente para asistir al personal de salud (particularmente médicos generales y especialistas) en la toma de decisiones sobre las acciones y procedimientos para la detección, diagnóstico y tratamiento del CaMa (guías y protocolos de práctica clínica). Estas iniciativas en su mayoría han contado con participación plural, interinstitucional e intersectorial para su elaboración y con la anuencia de las autoridades sanitarias del país para institucionalizarlas. En algunas ocasiones, se han adoptado a través de regulaciones y normativas de obligatorio cumplimiento para todo el sistema sanitario.

La mayoría de las guías y protocolos de práctica clínica establecen las acciones que se realizan tanto en el componente asistencial (técnico y clínico), como en el administrativo (competencias de cada nivel de atención, sistemas de referencia, mecanismos de información, monitoreo y vigilancia epidemiológica de los casos detectados). Un aspecto que llama la atención es la variabilidad de criterios para realizar estudios de mamografía: edad de inicio, periodicidad de la prueba, población a la cual se dirige. Sin embargo, un elemento común encontrado en la mayoría de los programas es que ante la sospecha de patología maligna se establecen los mecanismos para referir a las personas que presentaron anormalidades a pruebas de confirmación diagnóstica y en muchos casos a tratamiento.

\section{Discusión}

Antes de plantear la discusión y las conclusiones, es relevante establecer las limitaciones del estudio. La revisión documental, aunque aporta hallazgos significativos en la identificación de los marcos regulatorios y su contenido, los avances en el diseño y desarrollo de políticas y programas, así como de otros mecanismos para la detección temprana del cáncer de mama en AL, tiene limitaciones importantes por la imposibilidad de encontrar datos publicados de la totalidad de países de la región y sobre resultados de evaluaciones de las políticas y programas analizados. No se pudo establecer si se cumple o no el marco normativo establecido en cada país, si se están logrando los objetivos y metas planteadas en los programas, si éstos operan adecuadamente, ni si han logrado o no resultados y/o impacto en el acceso, oportunidad y calidad de la atención. Cabe señalar que no fue objeto de esta investigación medir el impacto de tales acciones. Sin embargo, actualmente el equipo de investigación está desarrollando una segunda fase bajo el patrocinio de la American Cancer Society para realizar un score card en cuatro países de la región que dé cuenta de los resultados y efectos de la implementación de políticas de atención al cáncer de mama en cada uno de estos países.

La literatura publicada establece que la detección temprana del CaMa y la garantía de un tratamiento integral tienen un efecto sinérgico para lograr un mejor desenlace de la patología en las personas. ${ }^{45-48}$

En países de ingresos altos, la implementación de acciones para la detección temprana han sido exitosas porque han estado basadas en proporcionar: a) un servicio universal para todas las personas sobre la base de necesidad de salud y no la capacidad de pago; b) acceso a una gama amplia de servicios de salud basada en la atención primaria, comunitaria y hospitalaria; c) servicios de información y apoyo basados en la promoción de la salud, la prevención de la enfermedad, el autocuidado y el mantenimiento de la salud. ${ }^{49-51}$ Esto ha implicado una política permanente de motivación y educación a las mujeres para realizarse el autoexamen mamario, hacer su examen clínico de mama y el desarrollo de programas de tamizaje poblacional organizado con mastografía. Si bien una detección y un diagnóstico temprano aportan significativamente a la reducción de la mortalidad por CaMa, su descenso no ha dependido exclusivamente de la efectividad de estos programas, sino también de la garantía de acceso a tratamiento oportuno y de calidad.

En los países de ingresos medios y bajos de AL, las estrategias de detección temprana han sido incorporadas dentro de las normas, reglamentos, leyes y programas. En estos últimos, el cáncer de mama se encuentra incorporado o se anida en programas más amplios de salud de la mujer o en programas preventivos. Ejemplo de ello se observa en países como Argentina, Brasil, Bolivia, Panamá, Perú, El Salvador y Colombia. Teniendo en cuenta las recomendaciones realizadas por la Iniciativa Global de Salud de la Mama (IBHI por sus siglas en inglés) para los países con recursos limitados, esta práctica es adecuada porque permite emplear los recursos y la infraestructura ya existentes y optimiza su utilización; sin embargo, la experiencia ha mostrado que en algunos de estos países este hecho ha generado competencia por recursos financieros dentro de un mismo programa, lo cual desfavorece su adecuada implementación y operación.

Un importante hallazgo derivado de la revisión muestra marcadas divergencias en la operación de programas, protocolos y guías de práctica clínica para la detección temprana y el diagnóstico oportuno del CaMa en la región. Cada una de las iniciativas desarrolladas presenta una denominación particular, el ámbito de aplicación es variable (nivel local/municipal, provincial/ estatal o nacional), los objetivos suelen ser muy especí- 
ficos de acuerdo a los lineamientos y estrategias de la política nacional en los que se insertan, con poblaciones disímiles a las que van dirigidas sus acciones (mujeres de 30 a 50 años) y marcadas diferencias en los criterios para los estudios mamográficos, particularmente en relación a la edad de inicio, frecuencia de aplicación y número de proyecciones.

Incluso dentro de un mismo país, a partir de su organización político-administrativa, de las condiciones económicas, sociales y epidemiológicas de cada sub-región y de la segmentación del sistema de salud, * coexisten diversos programas de detección de CaMa, la mayoría de las veces sin ningún tipo de articulación. Las implicaciones de esta multiplicidad de programas en un mismo país son muy variadas: a) duplicación de esfuerzos y recursos; b) incremento en los costos; c) desequilibrio y asignación insuficiente de recursos; d) concentración de actividades en grupos de población y áreas geográficas determinadas, e) heterogeneidad y potencial inequidad en el acceso a servicios de detección temprana, diagnóstico y tratamiento; f) normatividad diferente para cada institución; g) discrepancia en los sistemas de información.

En lo que se refiere a los programas de tamizaje, si bien las normativas establecen el uso de la mamografía como una acción para la detección temprana, estos se hacen como programas de tamizaje de oportunidad y no como tamizaje poblacional organizado. Un claro ejemplo de esta situación se presenta en Brasil y Colombia ${ }^{52,53}$ en donde se ha señalado la inconveniencia de implementar este tipo de programas aduciendo la escasez de recursos económicos para garantizar, una vez detectada la patología, la confirmación diagnóstica, el acceso a tratamiento oportuno y de calidad, y el seguimiento para todas las mujeres, particularmente, las más pobres $\mathrm{y} \sin$ aseguramiento en salud.

\section{Conclusiones}

Al tomar en cuenta algunas de las diversas lecciones de las experiencias exitosas de países que han logrado aciertos en la disminución de la mortalidad por cáncer

\footnotetext{
* Coexistencia de entidades públicas, de la seguridad social y de la iniciativa privada, con notables diferencias en el financiamiento, aseguramiento y prestación de servicios de salud, cada uno de ellos "especializado" en diferentes segmentos de la población de acuerdo con su inserción laboral, nivel de ingreso, capacidad de pago y posición social.
}

de mama (Reino Unido, Canadá, Japón, Australia y EUA, entre otros), se puede señalar que el principal reto de los países de AL es desarrollar programas integrales de atención de cáncer de mama, adecuadamente estructurados y con los recursos apropiados.

Específicamente en materia de detección temprana, debido a la escasez de recursos financieros en la región, no será efectivo implementar programas de tamizaje poblacional organizado si el sistema de salud no garantiza el acceso a confirmación diagnóstica, tratamiento y seguimiento a todas las mujeres que lo requieran independientemente de su capacidad de pago, porque de otra forma puede convertirse en un mecanismo más de inequidad en el acceso a los servicios de salud. Esto requerirá del diseño de programas de detección innovadores basados en estrategias educativas y motivacionales para las mujeres que garanticen el desarrollo de hábitos saludables como la autoexploración mamaria y el examen clínico de mama anual (realizado por personal bien entrenado y capacitado).

Ligado a lo anterior, otros de los desafíos a los que deberá enfrentarse la región para desarrollar políticas efectivas de detección temprana del CaMa son: a) lograr una mayor articulación interinstitucional e intersectorial entre las organizaciones gubernamentales y otros actores como las agremiaciones profesionales y las organizaciones de la sociedad civil; b) garantizar la aplicación de la normatividad y de los programas establecidos para la prevención secundaria del cáncer de mama en cada uno de los países de la región; c) consolidar sistemas de evaluación, monitoreo y seguimiento de los programas de prevención secundaria con el fin de conocer los resultados e impacto de su aplicación sobre la población afectada con la enfermedad; d) desarrollar la capacidad rectora de las autoridades sanitarias (la cual es diferente en cada país) para que cumplan un papel de coordinación en la movilización de recursos con miras a mejorar la equidad en el acceso a la atención, particularmente de las mujeres de menores recursos económicos.

La ejecución de las políticas es sin duda la gran prueba de puesta en operación del marco normativo. Aquí se requiere de una gran voluntad para movilizar recursos financieros, tecnológicos y humanos. Por tanto, una política sin una planeación adecuada de la participación de los actores involucrados, públicos y privados y una definición presupuestaria precisa, difícilmente tendrá un futuro exitoso.

\section{Agradecimientos}

Los autores agradecen el apoyo financiero y técnico del Instituto Carlos Slim de la Salud; de la iniciativa Cáncer 
de Mama: Tómatelo a Pecho, de la Fundación Mexicana para la Salud y su Consejo Promotor Competitividad y Salud; y del Consejo Nacional de Ciencia y Tecnología (CONACyT), convenio 85055, del Fondo Sectorial de Investigación para la Educación.

También agradecemos de manera especial a la doctora Felicia Knaul por ser inspiradora del esfuerzo que llevó a la generación de este producto, así como por apoyarlo en todo momento.

\section{Declaración de conflicto de intereses}

Declaramos no tener conflicto de intereses.

\section{Referencias}

I. Lozano-Ascencio R, Gómez-Dantés H, Lewis S, Torres-Sánchez L, López-Carrillo L. Tendencias del cáncer de mama en América Latina y El Caribe. Salud Publica Mex 2009;5I supl 2:SI47-SI56.

2. Robles SC, Galanis E. El cáncer de mama en América Latina y el Caribe. Rev Panam Salud Publica [serial en Internet]. 2002 Aug [Consultado 9 abril 2009 ] ; | 2(2): | 4 |- | 143. Disponible en: http://www.scielosp.org/scielo. php?script=sci_arttext\&pid=S1020-498920020008000 I $6 \&$ Ing=en. doi: I0.1590/SI020-49892002000800016

3. Lewis J.Análisis de la situación del Cáncer Cervicouterino en América Latina y el Caribe. Washington, DC: OPS, 2004.

4. Knaul F, López-Carrillo L, Lazcano E, Gómez-Dantés H, Romieu I,Torres G. Cáncer de mama: un reto para la sociedad y los sistemas de salud. Salud Publica Mex 2009; 5 I (2): I38-140

5. Palacio-Mejía LS, Lazcano-Ponce E,Allen-Leigh B, Hernández-Ávila M. Diferencias regionales en la mortalidad por cáncer de mama y cérvix en México entre 1979 y 2006. Salud Publica Mex 2009;5I supl 2:S208-S2I9. 6. Bosetti C, Malvezzi M, Chatenoud L, Negri E, Levi F, La Vecchia C. Trends in cancer mortality in the Americas, 1970-2000. Ann Oncol 2005; 16(3):489-5II.

7. Donoso SE, Cuello FM. Mortalidad por cáncer en la mujer chilena: análisis comparativo entre los años 1997 y 2003. Rev. chil. obstet. ginecol. [revista en la Internet]. 2006 [Consultado 25 febrero 2009 ] ; 71 (I): 10-16. Disponible en: http://www.scielo.cl/scielo.php?script=sci arttext\&pid=S07|7-75262006000 I00003\&lng=es. doi: $10.4067 /$ S07 I 7 75262006000100003

8. Knaul F, Nigenda G, Lozano R,Arreola-Ornelas H, Langer A, Frenk J. Breast cancer in Mexico: a pressing priority. Reprod Health Matters 2008 Nov; 16(32): I I3-23.

9. Martínez-Montañez OG, Uribe-Zúñiga P, Hernández-Ávila M. Políticas públicas del cáncer de mama en México. Salud Publica Mex 2009;5I supl 2:S350-S360

10. Secretaría de Salud. Subsecretaría de Prevención y Promoción de la Salud. Programa de Acción Específico 2007-2012: Cáncer de mama.

II. Kosters JP, Gotzsche PC. Regular self-examination or clinical examination for early detection of breast cancer. [Systematic Review] Cochrane Breast Cancer Group Cochrane Database of Systematic Reviews. 3, 2008

12. Barton MB, Fletcher SW, Harris R. Clinical breast examination for detecting breast cancer. JAMA 2000;283(13):1687-1689.

13. Miller AB, Baines CJ,Wall C. Canadian National Breast Screening Study-2: | 3 -year results of a randomized trial in women aged $50-59$ years. J Natl Cancer Inst 2000;92:1490-1499.
14. International Agency for Research on Cancer Working Group on the Evaluation of Cancer Preventive Strategies. Efficacy of screening by breast self examination. In:Vaionio H, Bianchini F, eds. Breast cancer screening. Lyon: IARC Press, 2002:107-1 I3

15. Sallerasa L, Domínguez A, Forés MD. Los métodos de la medicina clínica preventiva (III). Cribados. En: Med Clin (Barc) 1994; I02 Supl: 26-34. 16. Generalitat Valenciana. Conselleria de Sanitat. Programa de prevención de cáncer de mama en La ComunidadValenciana. Monografía Sanitaria Serie E No. 25. España, 1998.

17. International Agency for Research on Cancer. Breast Cancer Screening. IARC handbooks of Cancer Prevention, Vol 7. Lyon: IARC, 2002.

18. Anderson BO, Braun S, Lim S, Smith RA, Taplin S, Thomas DB. Early Detection of Breast Cancer in Countries with Limited resources. Breast J 2003;9(Supp12):S5I-59.

19. Smith RA, Saslow D, Sawyer KA, Burke W, Costanza ME, Evans WP 3rd et al. American Cancer Society High-Risk Work Group;American Cancer Society Screening Older Women Work Group;American Cancer Society Mammography Work Group;American Cancer Society Physical Examination Work Group;American Cancer Society New Technologies Work Group;American Cancer Society Breast Cancer Advisory Group. CA Cancer J Clin 2003; 53(3): |41-69.

20. Cox b. variation in the effectiveness of breast screening by year of follow-up.J Natl Cancer Inst Monogr 1997;69-72.

21. Glasziou P, Irwig L.The quality and interpretation of mammographic screening trials for women ages 40-49.J Natl Cancer Inst Monogr 1997; 73-77.

22. Kerlikowske K, Grady D, Rubin sm, Sandrock C, Ernster VI. Efficacy of screening mammography. a metaanalysis. JAMA 1995; 273: I49-I54. 23. Humphrey LI, Helfand M, Chan Bk, Woolf Sh. Breast cancer screening: a summary of the evidence for the U.S. preventive services task force. Ann Intern Med 2002; 137: 347-360

24. Ministerio de Sanidad y Consumo - Instituto de Salud "Carlos III" Agencia de Evaluación de Tecnologías sanitarias (AETS). Cribado Poblacional de Cáncer de Mama mediante Mamografía. Madrid:AETS Instituto de Salud Carlos II, Diciembre de 1995.

25. Sussman HR. Mammography screening for breast cancer in women under 50 years. Family Practice 2000; 17: 272-275.

26. Nyström L,Andersson I, Bjurstam N, Frisell J, Nordenskjold B, Rutgvist LE. Longterm effects of mammography screening updated overview of the Swedish randomized trials. Lancet 2002;359:909-919.

27. Legislatura de la Ciudad Autónoma de Buenos Aires. LEY N ${ }^{\circ} 2.960$ Sanción: 04/I2/2008 Promulgación: De Hecho del I3/0I/2009. Publicación: BOCBA N $^{\circ} 3106$ del 29/0I/2009. [Consultado I5 febrero 2009] Disponible en: http://www.cedom.gov.ar/es/legislacion/normas/leyes/ ley $2960 . \mathrm{html}$

28. Gobierno de Argentina. Ley 25673/2002. Programa Nacional de Salud Sexual y Procreación Responsable, en el ámbito del Ministerio de Salud. Buenos Aires, octubre de 2002. [Consultado I5 febrero 2009] Disponible en: http://test.e-legis-ar.msal.gov.ar/leisref/public/search. php? number $=25673 \mathrm{ta}$

29. Ministerio da Saúde. Secretaria de Assistência a Saúde. Instituto Nacional de Câncer.Viva Mulher. Programa Nacional de Controle do Câncer do Colo do Útero e de mama. Câncer do Colo do Útero: Informações Técnico Gerenciais e Ações Desenvolvidas. Rio de janeiro: INCA, 2002.

30. Ministerio de Salud y Deportes. Prevención y control de enfermedades no transmisibles en la atención primaria de salud: plan nacional $2005-$ 2009. / Ministerio de Salud y Deportes. La Paz: OPS/OMS, 2004. 3I. Masetti D, Sosa-Basaistegui M, Suarez-Hinojosa L,Terradas ML. Manual de Oncología para el Primer Nivel de Atención. Montevideo: Ministerio de Salud Pública, 2008.

32. Ministerio de Salud. Programa de Atención integral del Adulto y Adulto Mayor.Acuerdo Ministerial $N^{\circ} 0000153$. [Consultado I3 febrero 2009]. 
Disponible en: http://www.msp.gov.ec/index.php?option=com_content\&ta $s k=$ category\&sectionid $=8 \& i d=58 \& \mid$ temid $=116$

33. Ministerio de Salud Pública. Conjunto de Prestaciones del Sistema Nacional de Salud. $4^{a}$ Edición. Quito, Ecuador: Ministerio de Salud Pública, 2007.

34. Coalición Multisectorial "Perú contra el Cáncer". Plan Nacional para el Fortalecimiento de la Prevención y Control del Cáncer en el Perú. Lima, 2006. 35. Ministerio de Salud Pública y Asistencia Social. Guía Técnica De Prevención y Control Del Cáncer De Mama. El salvador: Ministerio de Salud Pública y Asistencia Social, 2006.

36. Ministerio de Salud de la provincia de Catamarca. Programa Móvil Rural de Detección Precoz Del Cáncer Génito-Mamario. Catamarca Argentina, 2007. [Consultado 30 noviembre 2008] Disponible en: http:// www.catamarca.gov.ar/salud/ministerio/index.php?option=com_content\&t ask $=$ view\&id=29\&ltemid $=32$

37. LEY N 5773 Programa Provincial de Prevención y Detección

Precoz de Cáncer de Cuello de Útero y Mama. Programa de Asistencia Previsional (PAP), Gobierno de Mendoza República de Argentina, 1991. 38. Peralta O. Cáncer de Mama En Chile: Datos Epidemiológicos. Rev chil obstet ginecol 2002; 67(6):439-445.

39. Ministerio de Salud. Política Nacional De Salud 2002 - 2006. Costa Rica: Ministerio de Salud, 2003

40.Argentina. Poder Ejecutivo Nacional. Resolución 93 del 9 de octubre del 2000. Programa Médico Obligatorio.

4I. Instituto Mexicano del Seguro Social. Cartilla de Salud y citas médicas. Mujeres de 20 a 59 años. México DF: IMSS, 2002.

42. Instituto Mexicano del Seguro Social. Guía para el cuidado de la salud. Mujeres de 20 a 59 años México DF: IMSS, 2002.

43. Consejo Nacional de Población. Informe de ejecución 200I-2003 del Programa Nacional de Población 200I-2006. México, DF: CONAPO, 2003 44. Secretaría de Salud. Programa de Acción 200 I-2006: Cáncer de Mama. México D.F: SS, 2002

45. Smith RA, Cokkinides V, Eyre HJ.American Cancer Society Guidelines for the Early Detection of Cancer, 2005. CA Cancer J Clin 2005;55:3 I-44. 46 Anderson, B, Yip C, Ramsey S, Bengua R, Bram S, Fitch M, et al. El cáncer de mama en los países con recursos limitados: Sistemas de Atención de Salud y Políticas Públicas. Grupo de Expertos sobre Atención de Salud y Políticas Públicas de la Cumbre Mundial de 2005, BHGl. Sistemas de Atención de Salud y Políticas Públicas. The Breast Journal 2007;13 Suppl I: S62-S82.

47. Jemal A, Murray T,Ward E, Samuels A, Tiwari RC, Ghafoor A, et al. Cancer statistics, 2005. CA Cancer J Clin 2005;55:10-30.

48. Gaudette LA, Silberberger C,Altmayer CA, Gao RN. Trends in breast cancer incidence and mortality. Health Reports. National Cancer Institute of Canada. Canadian cancer statistics, 1999. Toronto, Canada: National Cancer Institute of Canada, 1999:8(2):29-37.

49. Delivering Primary Care. (n.d.) [Consultado 04/03/2009] Disponible en: http://www.dh.gov.uk/AboutUs/DeliveringHealthAndSocialCare/ TheHealthAndSocialCareSystem/HealthAndSocialCareSystemArticle/fs/ en?CONTENT_ID $=4 \mid$ 05335\&chk=IRrIvj

50. Delivering Secondary Care. (n.d.) [Consultado 04/03/2009].

Disponible en: $h$ ttp://www.dh.gov.uk/AboutUs/DeliveringHealthAndSocialCare/ TheHealthAndSocialCareSystem/HealthAndSocialCareSystemArticle/fs/ en?CONTENT_ID $=4105336 \&$ chk $=v$ $\times$ Xz $9 n$

5I. Li S. Health Care Financing Policies of Canada, the United Kingdom and Taiwan. Research and Library Services Division Legislative Council Secretariat 2006

52. Gebrim LH, Quadros LGA. Rastreamento do câncer de mama no Brasil. Rev. Bras. Ginecol. Obstet. [serial on the Internet]. 2006 June [Consultado 28 abril 2009 ] ; 28(6): 319-323. Disponible en: http://www.scielo.br/scielo.php?script=sci_arttext\&pid=\$0 100 7203200600060000 I\&Ing=en. doi: $10.1590 / 50100-7203200600060000$ I.
53. Instituto Nacional de Cancerología. Recomendaciones para la tamización y la detección temprana del cáncer de mama en Colombia. Bogotá: INC, 2006

54. Olaviaga S, Maceira D. Mapa de actores del sector oncológico en la Argentina: objetivos, estrategias y mecanismos de articulación. Argentina: Centro de implementación de políticas públicas para la equidad y el crecimiento, 2007.

55. Legisalud Argentina. Programa Nacional de Salud Sexual y Procreación Responsable, en el ámbito del Ministerio de Salud. Ley 25.673 y decreto reglamentario I282/2003 (B. O. 26/05/2003). Buenos Aires: Legisalud Argentina 2002

56. Honorable Cámara de Diputados de la Nación. Reseña de Legislación Programa Médico Obligatorio. Legislación Nacional vigente. [Consultado 22 enero de 2009]. Disponible en: http://wwwl.hcdn.gov.ar/dependencias/ dip/documentos/RL.109.05.00-I.pdf.

57. Ministério de Saúde. Cadernos de Atenção Básica No. I3. Controle dos Cânceres do colo do útero e da mama. Brasília DF: Ministério de Saúde, 2006. 58. Ministerio de Salud de Chile. Ley N ${ }^{\circ} 19.966$ por la cual se establece el Régimen de Garantías en Salud. Septiembre 2004.

59. Ministerio de Salud de Chile. Listado de prestaciones especificas. Anexo al decreto supremo $N^{\circ} 44$ de 9 enero de 2007 de los Ministerios de Salud y Hacienda que establece Garantías Explicitas en Salud. 60. Ministerio de Salud Colombia. Resolución 526I por la cual se establece el Manual de Actividades, Intervenciones y Procedimientos del Plan Obligatorio de Salud en el Sistema General de Seguridad Social en Salud. Bogotá: Ministerio de Salud Colombia 1994.

61. Ministerio de Salud Colombia.Acuerdo 72 de 1997 por medio del cual se define el Plan de Beneficios del Régimen Subsidiado en el Sistema General de Seguridad Social en Salud. Bogotá: Ministerio de Salud Colombia, 1997.

62. República de Colombia. Ministerio de Salud. Resolución 4I2, febrero 25 de 2000, por la cual se establecen las actividades, procedimientos e intervenciones de demanda inducida y obligatorio cumplimiento y se adoptan las normas técnicas y guías de atención para el desarrollo de las acciones de protección específica, detección temprana y la atención de enfermedades de interés en salud pública. Bogotá: Imprenta Nacional, 2000 63. Diario Oficial de la Federación. Norma Oficial (NOM-04I-SSA2-2002) para la prevención, diagnóstico, tratamiento, control y vigilancia epidemiológica del cáncer de mama. México, DF: Diario Oficial de la Federación, 2003.

64. Ministerio de Salud. Caja de Seguro Social. Normas Técnico Administrativas y Manual de Procedimientos Programa de Salud Integral de la Mujer. Panamá: Ministerio de Salud, 2007

65. El Peruano. Diario Oficial. Resolución Jefatural N I20-RJ-INEN-2008 por la cual Aprueban la "Norma Técnico Oncológica para la Prevención, Detección y Diagnóstico Temprano del Cáncer de Mama a Nivel Nacional" publicado 22/04/2008. [Consultado 8 enero 2009]. Disponible en http://www.glin.gov/download.action?fulltextld=22806 I\&documentld= 21 2070\&glinlD $=212070$

66. Ministerio de Salud Pública Uruguay. Manual de oncología para el primer nivel de atención. [Consultado 2 febrero 2009] Disponible en: www.msp.gub.uy/andocasociado.aspx!2 103,15।35

67. Ministerio de Salud Provincia de Buenos Aires. Programa de prevención de cáncer génito-mamario (PROGEMA). [Consultado 8 enero 2009]. Disponible en: http://www.ms.gba.gov.ar/programas/progema/progema.html 68. Tobar F. Economía de la prevención. [Consultado 12 diciembre 2008]. Disponible en: http://www.federicotobar.com.ar/pdf/Economiadelasalud/ Economia-de-la-prevencion.pdf

69. Ministério da Saúde. Controle do Câncer de Mama. Documento de Consenso. Rio de Janeiro: Ministério da Saúde, 2004.

70. Prieto M. Programa Nacional De Cancer De Mama Chile, 1995 - 2009. Ponencia presentada en el Taller Internacional de tamizaje de Cáncer de 
Mama Río de Janeiro Brasil, 17 y 18 de Abril 2009. [Consultado 30 abril 2009]. Disponible en: http://bvsms.saude.gov.br/bvs/palestras/cancer/ programa_nacional_cancer_mama_chile.pdf

71. Instituto Mexicano del Seguro Social. Guía Técnica. Mujeres de 20 a 59 años. México DF: IMSS, 2002.

72. Ministerio de Salud, Caja de Seguro Social. Revisión de normas de salud integral de la mujer. Panamá: Ministerio de Salud, 2007.

73. Comisión Honoraria de Lucha contra el Cáncer (CHLCC). Programa de Detección Precoz de Cáncer de Mama. [Consultado 12 diciembre 2008]. Disponible en: http://www.urucan.org.uy/uilayer/dpcm/objetivos.htm 74. Academia Nacional de Medicina. Consenso Nacional Inter-Sociedades sobre Cáncer de Mama:Pautas para el Diagnóstico y Manejo de las Lesiones Mamarias Subclínicas. Argentina: Academia Nacional de Medicina, 2006.
75. Asociación Argentina de Ginecología Oncológica. Consenso Nacional Inter-Sociedades sobre Cáncer de Mama: Pautas para el seguimiento de pacientes asintomáticas luego del tratamiento primario con intención curativa. 2007. [Consultado I2 diciembre 2008]. Disponible en: http:// www.aaginonc.org.ar/articulos/consenso_seguimiento-pacientes_ asintomaticas_2007.pdf

76. Ministerio de Salud. Guía clínica de cáncer de mama en personas de 15 años y más. Santiago: Minsal, 2005.

77. Ministerio de Salud. Normas Y Procedimientos Para El Tratamiento Del Cáncer En Costa Rica. San José: Ministerio de Salud, 2009. 